\title{
Formation of Cavitation and Supercavitation in a Rectangular Shape Nozzle
}

\author{
Espanta Ferdowsian \\ Department of Mechanical Engineering \\ University of Maryland \\ USA
}

\begin{abstract}
Flow inside a rectangular shape nozzle is simulated in this study. Finite volume scheme is utilized as the main solver for the current study. Second order scheme is utilized to discretize pressure. Second order upwind scheme is utilized for solving momentum equation. Then the momentum equation is coupled with the continuity equation to obtain the pressure and velocity at each cell. Cavitation inception and super cavitation is also found and discussed in this study and the results were also verified with previous Winklhofer et al. experiments.
\end{abstract}

\section{Keywords: Cavitation; Multiphase; Turbulent; Flow; Mesh}

\section{INTRODUCTION}

Spray atomization is affected enormously by turbulence and cavitation in a diesel injector nozzle and then combustion performance is affected as well [1-5]. Cavitation has been investigated widely in real-size diesel injector nozzle even though there are many ambiguous questions remained unanswered. High fuel injection pressure and small-scale size of the nozzles makes it very hard to visualize the flow inside nozzles and therefore many simulations are necessary to be done in order to understand the process of cavitation. Experimental studies of cavitation due to complex nature of the mentioned phenomena are very limited to transparent models under a certain boundary conditions [6-12]. With development of CFD (Computational Fluid Dynamic) numerical simulation has been of interest in the recent years as it is faster and less expensive.

\section{NUMERICAL SCHEME}

Continuity and momentum equations for an incompressible fluid flow can be written as following [1, 4]:

$$
\begin{gathered}
\frac{\partial \bar{u}_{i}}{\partial x_{i}}=0 \\
\frac{\partial \bar{u}_{i}}{\partial t}+\bar{u}_{j} \frac{\partial \bar{u}_{i}}{\partial x_{j}}=-\frac{1}{\rho} \frac{\partial \bar{P}}{\partial x_{i}}+v \frac{\partial^{2} \bar{u}_{i}}{\partial x_{j} \partial x_{j}}-\frac{\partial}{\partial x_{j}} R_{i j}
\end{gathered}
$$

In which $\bar{u}_{i}$ is the mean velocity, $t$ the time, $x_{i}$ is the position, $\rho$ is the constant density, $\bar{P}$ is the mean pressure, $R_{i j}=\overline{u^{\prime}{ }_{l} u^{\prime}}{ }_{j}$ is the Reynolds stress tensor and $v$ is the kinematic viscosity and finally $u_{i}^{\prime}=u_{i}-\bar{u}_{l}$ is the fluctuating components of the velocity.

The RSTM evaluates differential transport equations in order to obtain turbulence stress components:

$$
\begin{aligned}
\frac{\partial}{\partial t} R_{i j}+\bar{u}_{k} \frac{\partial}{\partial x_{k}} & R_{i j} \\
& =\frac{\partial}{\partial x_{k}}\left(\frac{v_{t}}{\sigma^{k}} R_{i j}\right)-\left[R_{j k} \frac{\partial \bar{u}_{j}}{\partial x_{k}}\right] \\
& -C_{1} \frac{\epsilon}{k}\left[R_{i j}-\frac{2}{3} \delta_{i j} k\right] \\
& -C_{2}\left[P_{i j}-\frac{2}{3} \delta_{i j} \epsilon_{1}\right]
\end{aligned}
$$

In which the turbulent production can found from the following equations:

$$
P_{i j}=-R_{j k} \frac{\partial \bar{u}_{j}}{\partial x_{k}}-R_{j k} \frac{\partial \bar{u}_{i}}{\partial x_{k}}
$$

The turbulent dissipation rate is also calculated from the following equation:

$$
\begin{aligned}
& \frac{\partial \epsilon}{\partial t}+\bar{u}_{j} \frac{\partial \epsilon}{\partial x_{i}}= \frac{\partial}{\partial x_{i}}\left[\left(v+\frac{v_{t}}{\sigma^{\epsilon}}\right) \frac{\partial \epsilon}{\partial x_{i}}\right]-C^{\epsilon 1} \frac{\epsilon}{k} R_{i j} \frac{\partial u_{i}}{\partial x_{j}} \\
&-C^{\epsilon 2} \frac{\epsilon^{2}}{k}
\end{aligned}
$$

Instantaneous fluid velocity was also obtained from the following equation:

$$
\frac{d u_{i}}{d t}=-\frac{u_{i}-\bar{u}_{l}}{T_{I}}+\left(\frac{2{\overline{u_{l}^{\prime}}}_{l}^{2}}{T_{I}}\right) \xi_{i}(t)
$$

The average time in which the turbulent eddies spent time in a particle track is obtained as following:

$$
T_{I}=\int_{0}^{\infty} \frac{u_{p}^{\prime}(t) u_{p}^{\prime}(t+s)}{u_{q}^{\prime} u_{q}^{\prime}} d s
$$

Bubble trajectory has also been obtained from the following equations: 


$$
\begin{gathered}
R \ddot{R}+\frac{3}{2} \dot{R}^{2}=\frac{1}{\rho}\left[\left(P_{g}-P_{0}-P_{S}\right)-4 \mu \frac{\dot{R}}{R}-2 \frac{\sigma}{R}\right. \\
\left.+\frac{R}{c} \frac{d}{d t}\left(P_{g}\right)\right] \\
P_{g}(t)=\left(P_{g 0}\right)\left(\frac{R_{0}}{R}\right)^{3 \gamma} \\
P_{g 0}=P_{\text {inf }}+2 \frac{\sigma}{R_{0}}-P_{c r}
\end{gathered}
$$

Bubble motion equation has also been used in order to obtain location of each cell during the calculation period:

$$
\begin{aligned}
& \frac{d U_{b}}{d t}=2 g \vec{\jmath}-\frac{3}{\rho} \nabla \vec{p}+\frac{3}{4} \frac{C_{D}}{R}\left(\vec{U}-\overrightarrow{U_{b}}\right)\left|\vec{U}-\overrightarrow{U_{b}}\right| \\
&+ \frac{3}{R}\left(\vec{U}-\overrightarrow{U_{b}}\right) \dot{R}+\frac{1.542}{R} v^{0.5}(\vec{U} \\
&\left.-\overrightarrow{U_{b}}\right)\left|\frac{d \vec{U}}{d y}\right|^{0.5} \operatorname{sgn}\left(\frac{d \vec{U}}{d y}\right) \vec{\jmath} \\
& C_{D}=\frac{24}{R e}\left(1-0.197 R e^{0.63}\right)+\frac{24}{R e}(2.6 \\
&\left.\times 10^{-4} \operatorname{Re}^{1.38}\right)
\end{aligned}
$$

\section{RESULT AND DISCUSSION}

At the beginning a structured dominant mesh is used in this study which is following the measurements introduced by Winklhofer et al. [13]. Figure 1 shows structured dominant mesh used in this study in which a fully structured mesh is used in the orifice area of the nozzle. Moreover, figure 2 shows close up view of the mesh near the orifice inlet.

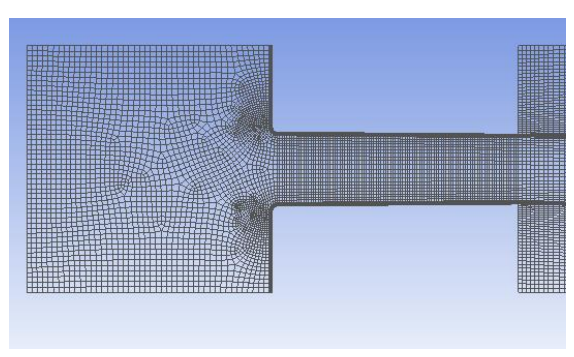

Figure 1. Mesh topology of the domain.

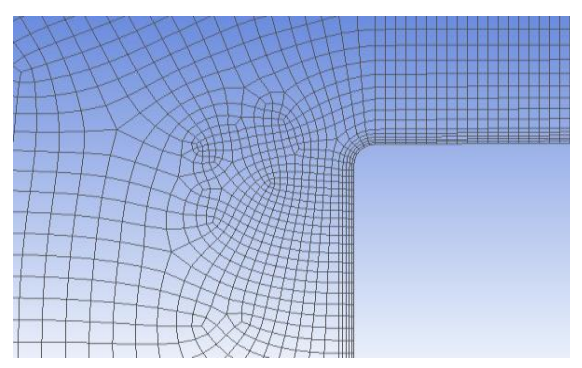

Figure 2. Close up view of the mesh near the orifice inlet.

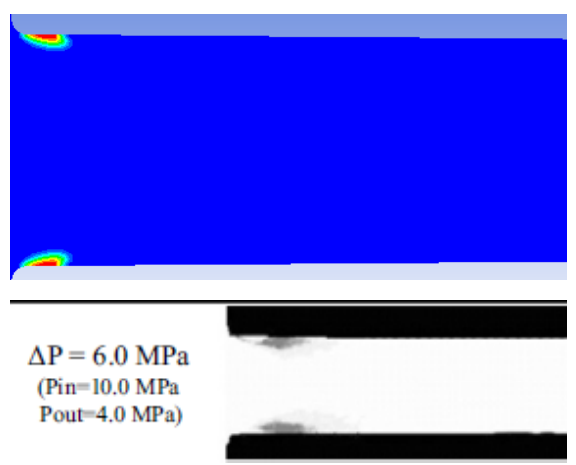

Figure 3. Contour of vapor volume fraction when cavitation inception occurs.

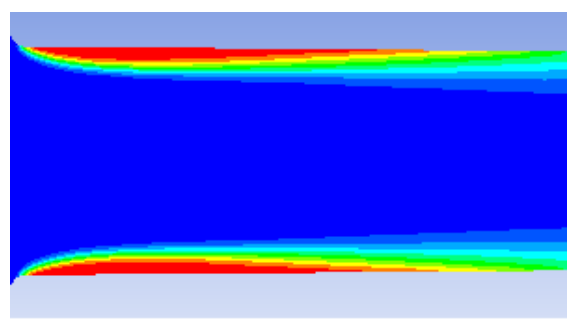

Figure 4. Contour of vapor volume fraction when super cavitation occurs.

As mentioned earlier the flow is solved using finite volume scheme where the two equations of continuity and momentum were solved together in order to obtain the velocity and pressure profile. Then using a structured dominant mesh as shown in figure 1 and 2 the whole domain is divided in to smaller domains to make it feasible for utilization of the current numerical scheme.

Figure 3 and 4 shows formation of cavitation at two stages which are namely inception and final. During 
the cavitation inception in which the inlet pressure is fixed to $10 \mathrm{MPa}$ and the outlet pressure is fixed to 4.0 MPa, only a smaller region of cavitating region forms which leads to affect the inlet radius region. It can also be seen from figure 3 that the inception region predicted from the simulation is matching with previous experimental result shown in black and white.

Finally, figure 4 shows formation of super cavitation when the inlet pressure is fixed to $10 \mathrm{MPa}$ and the outlet pressure is fixed to $2.5 \mathrm{MPa}$. It can be seen that the whole orifice area is covered by the vapor volume fraction in this case and any further increase in the pressure difference would make the flow choke and the whole physical properties would change.

\section{CONCLUSION}

In this study the flow is simulated in a rectangular shape nozzle using finite volume scheme. In order to make the simulation process feasible the domain is divided in to smaller regions using structured dominant mesh.

1- Cavitation inception was found and verified using previous experimental contour.

2- Super cavitation was found and verified using previous experimental contour.

\section{REFERENCES}

[1] SMJ Zeidi, M.M., Numerical investigation of the effect of different parameters on emitted shockwave from bubble collapse in a nozzle. Journal of Particle Science \& Technology, 2021. 6(2): p. 13.

[2] Zeidi, S. and M. Mahdi, Investigation the effects of injection pressure and compressibility and nozzle entry in diesel injector nozzle's flow. Journal of Applied and Computational Mechanics, 2015. 1(2): p. 83-94.

[3] Zeidi, S. and M. Mahdi. Investigation of viscosity effect on velocity profile and cavitation formation in Diesel injector nozzle. in Proceedings of the 8th international conference on internal combustion engines. 2014.

[4] Zeidi, S.M.J. and M. Mahdi, Evaluation of the physical forces exerted on a spherical bubble inside the nozzle in a cavitating flow with an Eulerian/Lagrangian approach. European Journal of Physics, 2015. 36(6).

[5] SMJ Zeidi, M.M., Effects of nozzle geometry and fuel characteristics on cavitation phenomena in injection nozzles. Proceedings of the 22st Annual International Conference on Mechanical Engineering-ISME, 2015.

[6] Azadeh Yazdi, M.N., Sepideh Amirahmadian, Nasim Sabetpour, Amirmasoud Hamedi, Utilization of SchnerrSauer Cavitation Model for Simulation of Cavitation Inception and Super Cavitation. International Journal of Aerospace and Mechanical Engineering, 2021. 15(7).

[7] Mohammadreza Nezamirad , S.A., Nasim Sabetpour ,Amirmasoud Hamedi ,Azadeh Yazdi, Effect of Needle Height on Formation of Cavitation in a Six-Hole Diesel Injector Nozzle. 6th national conference on Mechanical and Aerospace Engineering, 2021.

[8] Mohammadreza Nezamirad, N.S., Azadeh Yazdi, Amirmasoud Hamedi, Investigation the Effect of
Velocity Inlet and Carrying Fluid on the Flow inside Coronary Artery. International Journal of Aerospace and Mechanical Engineering, 2021. 15(7).

[9] Nasim Sabetpour, A.Y., Sepideh Amirahmadian, Mohammadreza Nezamirad, Amirmasoud Hamedi, Formation of Vapor Volume Fraction in a real size nozzle using Schnerr and Sauer approach. Forth Conference on Technology Development in Mechanical and Aerospace Engineering, 2021.

[10] Mohammadreza Nezamirad, S.A., Nasim Sabetpour, Azadeh Yazdi, Amirmasoud Hamedi, Effect of Needle Height on Discharge Coefficient and Cavitation Number. International Journal of Aerospace and Mechanical Engineering, 2021. 15(7).

[11] Mohammadreza Nezamirad, S.A., Nasim Sabetpour, Azadeh Yazdi, Amirmasoud Hamedi, Effect of Needle Height on Discharge Coefficient and Cavitation Number. International Journal of Aerospace and Mechanical Engineering, 2021. 15(7).

[12] Mohammadreza Nezamirad, S.A., Nasim Sabetpour, Azadeh Yazdi,Amirmasoud Hamedi, Effect of Different Diesel Fuels on Formation of the Cavitation Phenomena. International Journal of Aerospace and Mechanical Engineering, 2021. 15(7).

[13] Winklhofer, E., et al. Comprehensive hydraulic and flow field documentation in model throttle experiments under cavitation conditions. in Proceedings of the ILASSEurope conference, Zurich. 2001 